\title{
Visualization of Electric Discharge in Porous Materials
}

\author{
A. Elzowawi, A. Haddad, H. Griffiths \\ Advanced High Voltage Engineering Research Centre, Cardiff University \\ The Parade, Cardiff, UK \\ ElzowawiA@cardiff.ac.uk
}

\begin{abstract}
Very few studies have been conducted to visualize the dynamic discharge development from ground electrode in the soil. The imaging process of the electric discharge in opaque porous materials is extremely difficult. Therefore, in this study, a photographic investigation of the electric discharge in a new dielectric glass bubble material was conducted. A Photron FASTCAM SA5 high speed camera was utilized to record the discharge light emitted from the sample.

A special test rig was developed to facilitate these tests with a rod - plane electrode configuration. The material was placed in a transparent acrylic tube to allow the discharge light to be transmitted so that the camera can record the discharge. Standard lightning and switching impulse voltages were used in these experiments. Synchronized frames of the recorded video with voltage and current waveforms allow visualization of the discharge phenomenon and monitoring the dynamic change of the discharge at various times during the impulse.
\end{abstract}

\section{INTRODUCTION}

The soil ionization phenomenon is considered as one of the main causes for nonlinear behaviour observed in the impedance of earthing systems under high currents such as those generated by lightning impulses. Despite of the attention that the ionization phenomenon has drawn and the large number of experiments that has been published, it is still ambiguous and not fully understood. Most of the research investigations examined this phenomenon in terms of the electrical aspect, such as electric field, current density, resistivity, and by measuring and recording the voltage and the current waveforms. However, studying the electric discharge visually in the soil was found to be a good auxiliary element to have a better understanding of the phenomena and to provide new information on the development and the shape of the discharge inside the soil.

The imaging process of the electric discharge in porous materials like soils is extremely difficult, which could be due (a) the physical properties in terms of color, size and shape of the grains, and water content, (b) the chemical and electrical nature of the material in terms of the chemical composition, permittivity and conductivity, (c) the discharge mechanism in the porous material where the soil ionization phenomenon could occur, (d) the type, shape and polarity of applied voltage. In the literature, techniques such as x-ray films[1-5], photographic films[3] and conducting paper[6], have been used to record the electric discharge and the ionization phenomena from inside the soil, by inserting film materials near the electrodes within the soil sample. These, techniques are simple, cheap, easy to use, and they already have given very useful images which have helped with understanding the phenomena, especially x-ray film which is considered as the most commonly used technique in this field. However, these methods suffer from a few disadvantages; for instance, they give a restricted view of the discharge. Moreover, they cannot capture the dynamic change of the discharge with time. Besides, in the case of strong discharges, the film will suffer from punctures and color distortion, due to the thermal and light sensitivities.

The purpose of this paper is to image the electric discharge in a new dielectric porous material (Glass Bubbles), using a different technique from those in the literature, i.e. using a high speed camera; so that the whole discharge process can be recorded and analyzed in conjunction with the voltage and current waveforms. Thus, the recorded videos show the development of the discharge process with time.

\section{GLASS BUBBLES RESISTIVITY TEST}

The glass bubble material consists of hollow low density glass microspheres (average diameter is 30 microns). It is a white powder and an insoluble substance, which is made from soda lime-borosilicate glass. These microspheres are used as an additive in many industrial applications to improve the properties of various materials and compounds. Due to the lack of the electrical resistivity value of the glass bubble material in any available source, a resistivity measurement test was performed to determine the resistivity of glass bubbles S38XHS for the first time. A parallel plate configuration was used to measure the resistance of the dry material placed between the two electrodes, and the resistivity of the material was calculated.

The test circuit includes ABEM SAS1000 tester, and the test cell which consists of two parallel square aluminum plates $(20 \mathrm{~cm} \times 20 \mathrm{~cm})$. These two electrodes are fixed inside a special container. Dry glass bubble material was poured between the two electrodes to completely fill the gap between them. The tester was connected with the electrodes to inject a DC current into the material. Therefore, using the available test equipment, a resistance value was obtained from $1 \mathrm{~mA}$ current injection. From the dimensions of the plates and the distance between them, the resistivity was calculated. 
Plate area $(\mathrm{A})=0.2 \mathrm{~m} \times 0.2 \mathrm{~m}=0.04 \mathrm{~m}^{2}$

The distance between the plates $(\mathrm{L})=0.04 \mathrm{~m}$

The measured resistance value from the tester $(\mathrm{R})=325 \mathrm{k} \Omega$

$$
\rho=\frac{R \times A}{L}=325 \Omega m
$$

It can be concluded that the DC resistivity of the glass bubble material is $325 \mathrm{k} \Omega \mathrm{m}$. Note that the test was performed in the normal laboratory atmospheric conditions (temperature $21 \mathrm{C}^{\circ}$, pressure 1 bar, RH 63\%) and the test was repeated 4 times.

\section{TEST ARRANGEMENTS}

\section{A. Test setup:}

Fig. 1 shows a special test rig which was developed to facilitate these tests with rod to plane electrode configuration. The material was placed in a transparent tube $(8 \mathrm{~cm}$ diameter and $50 \mathrm{~cm}$ height) to allow the discharge light to be transmitted through it so that the camera can record the discharge. For the lightning impulse voltage, a Haefely impulse voltage generator with four stages which can generate up to $400 \mathrm{kV}$ was used. For the switching impulse voltage, a Ferranti impulse voltage was reconstructed with four stages to generate a switching voltage. The high speed camera was operated at a high frame rate to record the discharge in microseconds. A capacitive voltage divider with a ratio 27931:1 and current transformer with a sensitivity 10:1 V/A were used to measure the voltage and current signals. These signals were captured on a Lecroy Wavejet 314 digital oscilloscope. The test circuit is shown in Fig. 2.

\section{B. Sample preparation:}

Due to the high resistivity of this material, the dry glass bubble material tests did not show any ionization, and no conduction current was detected until the full breakdown occurred. The breakdown path was observed at the interface between the glass bubbles and the inner surface of the tube and not in the middle of the material. Therefore, to make sure that the whole discharge occurs inside the material, and to obtain conduction current even before breakdown, and also to have some control on the current propagation path to direct it to the side where the camera is, the sample was sectioned into two parts, the upper part will be just dry glass bubbles where the discharge will take place and the lower part was divided into two further subsections.

The subsection that faces the camera contained wetted glass bubble material with tap water, and the other or the back subsection was filled with dry material. This new arrangement has shown significant improvements to the test, where the current now flows towards the less resistive section which is that facing the camera (wetted part). To ensure a good moisture distribution among the grains, the material and water were thoroughly mixed. Then, this mixture is poured into the lower part of the tube until reaches a specific height. At this point, some pressure was manually applied on the sample to make sure that the mixture is suitably compacted and distributed uniformly in the tube, which prevents formation of gaps inside the sample. The dry material was then poured over the wet material.

\section{LIGHTNING IMPULSE TESTS}

Lightning impulse voltages were applied to the sample. An instantaneous triggering system of the impulse generator and the high speed camera was developed to record the whole discharge process in the dry glass bubbles. The high speed camera was setup to operate at 175000 frames per second, so that the camera can have a suitable window to visualize the whole dry material part. Setting the camera at that frame rate will produce frames with $5.5 \mu$ s duration each.

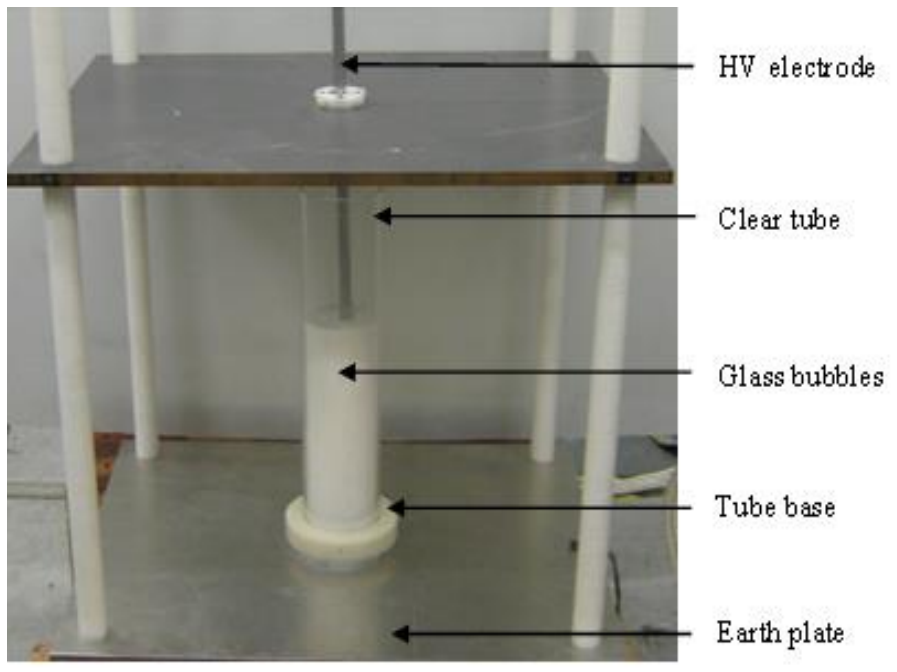

Figure 1. Test cell

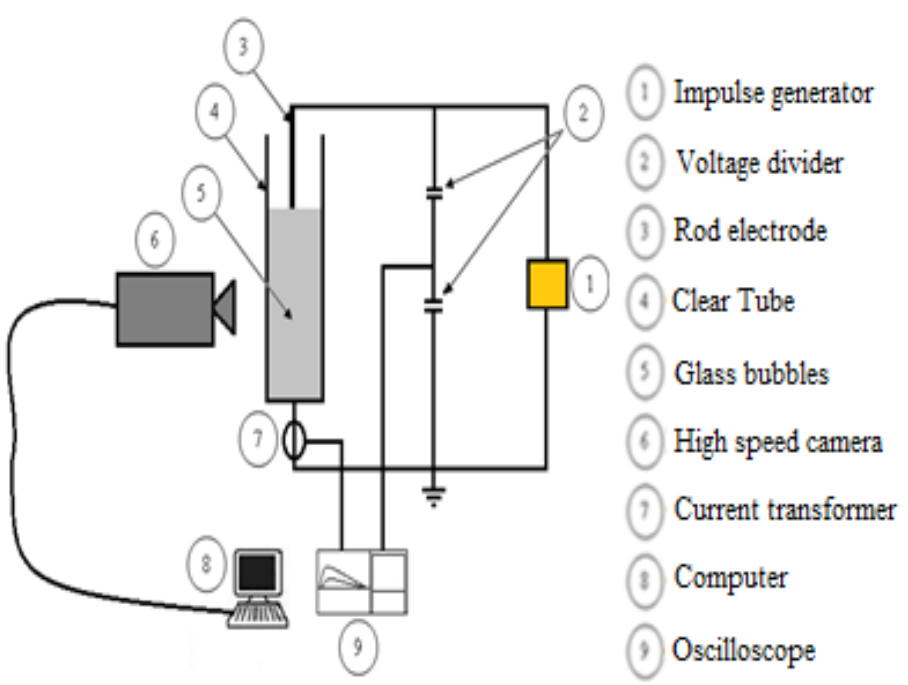

Figure 2. Test circuit

A. Discharge without a Breakdown:

Due to the streamers, flashing light could be seen around the electrode during the test without detecting current. At a certain voltage level when the electric field is high enough, current flow from active electrode to the ground was detected by both the current transformer and the camera; whereas the voltage 
did not show any sign of breakdown. The current stream in the dry material could be due to the ionisation in the air pockets among the dry microspheres, which could support the ionisation phenomenon in dry soil. By processing the recorded video, it was observed that the intensity of the emitted discharge light depends on the current magnitude, so the brightest light was occurring at the current peak, and as the current decays the light intensity decreases as well. On the other hand, by observing the current waveform, there was a time delay before the current started to increase, which could indicate the time for the ionisation phenomenon to develop in the air voids and for the streamer to cross the dry high resistivity material. With the increase of the applied voltage, this delay time was reduced. With the recorded video was synchronised with measured voltage and current waveforms, video frames from the high speed camera were paired with specific instants on the voltage and current impulse traces. Fig. 3 shows an example of synchronised current and voltage waveforms with particular frames. Each horizontal division in the figure is $5.5 \mu \mathrm{s}$, and each video frame is also $5.5 \mu \mathrm{s}$. Each frame is associated with the corresponding time division of the electrical signals, and the light intensity in each frame captures the discharge in this time range. The height of the frame represents the dry material part, and frame width represents the tube diameter. Furthermore, the location of the electrode tip is at the top of each frame.

\section{B. Discharge with a Breakdown:}

By increasing the applied voltage level, conduction current was also observed. The current started with a low value and then developed to a breakdown with higher current magnitude and lower voltage. The occurrence of the breakdown may be due to the growth and development of the ionization zone to reduce the resistivity even more to allow more current to flow. At the onset of the ionisation, a very faint light can be seen from the terminal electrode. This then developed at the maximum ionisation to form a very bright arc representing the breakdown. As the current dropped, the deionisation process was taking place with a longer time compared with the previous case (Fig 3). The delay time was also less than that in the previous case. Fig. 4 shows the synchronisation between the video frames and the voltage and current waveforms.

\section{SWITCHING IMPULSE TESTS}

With the same procedures as for the lightning tests, switching impulse voltages were applied onto similar sample settings. The high speed camera was setup to operate at 100000 frames per second, so that the camera at this frame rate will produce frames with $9.8 \mu \mathrm{s}$ each. Switching voltages were applied up to a level where a current was detected by the current transformer, and also recorded by the camera. The current conduction lasted for a longer time due to the longer impulse voltage, and this has led to longer recorded videos duration. It was also observed that when the current increased, the voltage dropped to a certain value until the current approached zero, and then voltage recovery occurred. Fig. 5 shows the synchronized video frames with the voltage and current waveforms. Due to the large number of frames, only the important frames were considered as numbered in the figure. With regard to the video of this discharge, it showed similar behaviour to the lightning discharge at the beginning of the current flow, so the brightness of the discharge light was corresponding to the current value, until about $215 \mu$ s, frame number 22 , when the current was decreasing; an increase in the brightness of the light was witnessed. This was different from the lightning discharges, where the period of the current flow did not exceed $200 \mu$ s even in the breakdown case. To explain this new phenomenon, more tests should be conducted and new parameters should also be considered such as the electron density and the temperature. However, an initial comment, relating to the investigation in [7], is that the increase in the light brightness might be due to the increase in the electron density which led to an increase in the plasma.

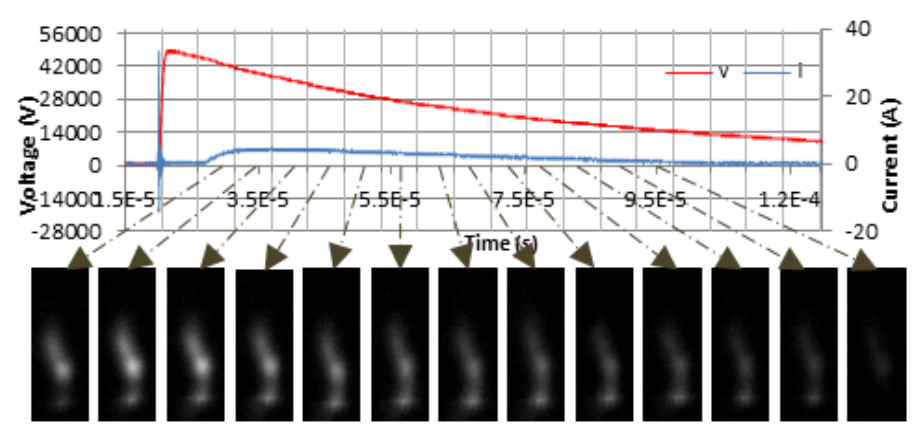

Figure 3. Synchronised video frames with voltage and current waveforms in the case of no breakdown

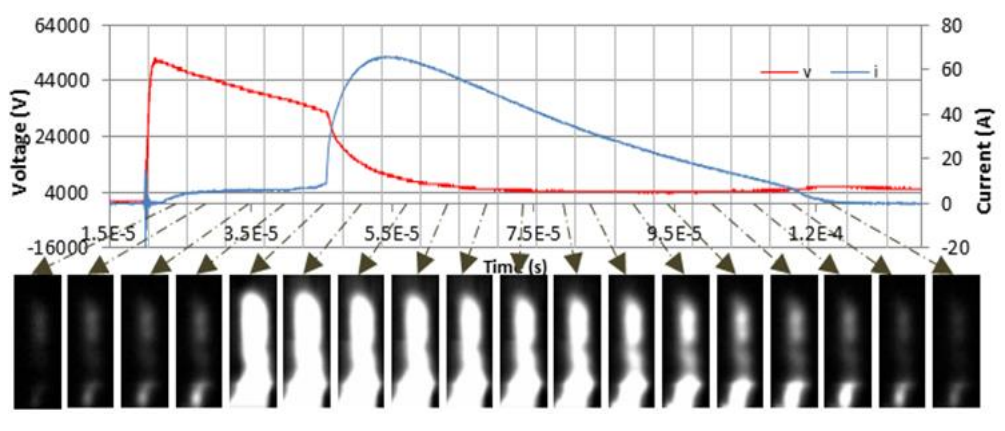

Figure 4. Synchronised video frames with voltage and current waveforms in the case of breakdown

\section{OBSERVATIONS AND DISCUSSION}

Close examination of the discharge channels in the obtained videos revealed that the current propagates in the direction of the electric field lines, which starts from the surface of the active electrode towards the ground plate, in a vertical manner with only one single channel. Due to the dependence of the camera on the emitted light, it is not possible to record every discharge with the high speed camera. This could be due to the uncontrollable discharge behaviour and the discharge propagation path. The light was produced by the flow of the current through an ionized path i.e. ionized air voids among the glass bubbles. Therefore, when the electric field is high enough to ionize the air voids among the glass microspheres, the ionization phenomenon takes place around the active electrode and then extends towards the ground. If the ionization zone reaches the wet part of the sample, then the current will flow. This process is thought to be causing the delay time at the start 
of the current trace. Hence, the ionization reaches its maximum at the current peak. After that, when the current starts to decrease, the deionization process begins until the current approaches zero. However, in the case of breakdown, different scenarios could occur. For instance, due to the higher electric field, the ionization process will occur faster, so the resistivity of the dry section will drop to allow the current to flow. The other scenario is that the resistivity of the wet material drops as well due to either thermal or electrical effects, in turn might cause a decrease in the resistivity of the current path leading to a breakdown.

The discharge under switching voltage has a similar behaviour, but it has a longer time delay compared with the lightning discharge, where the voltage impulse has a slower rise time. The discharge channels have very small diameters according to the diameters of the holes found in the wet region, not as they appear in the videos.

\section{CONCLUSIONS}

The laboratory resistivity tests of the dielectric glass bubble material have revealed a high resistivity value of this material. Ionization and breakdown phenomena were recorded and visualized in the high resistivity dry glass bubbles. Moreover, synchronized frames of the recorded video with voltage and current waveforms allowed visualization and monitoring of the discharge phenomena at various times during the impulse. Similar results were obtained with lightning and switching voltages. Furthermore, using the Glass bubble material and the high speed camera has given wider and different views of the discharge phenomena and showed the dynamic change of the discharge development. Therefore, these results of this study could help to clarify the process of soil ionization in earthing systems.

\section{REFERENCES}

[1] Hayashi M.," Observation of Streamer in the Soil by Surge Current", The Journal of the Institute of Electrical Engineers of Japan, Vol. 87, no. 940, pp. 133-141, 1967.

[2] Víctor M. and Cabrera M., "Photographic investigations of electric discharges in Sandy media", Journal of Electrostatics, Vol. 30, no. 0: pp. 47-56, 1993.

[3] Han X., Peng M.,Pu B.,Xia C.,Yu J.,Wang Y.,Zhou Wand Hu M., "The experimental study of spark breakdowns in soil", Power Engineering and Automation Conference (PEAM), Vol. 2, pp. 36-40, 2011.

[4] He J., Yuan J., and Zhang B., "Photographic Investigations on Lightning Impulse Discharge Phenomena in Soil", IEEJ Transactions on Power and Energy, Vol. 133, no. 12: pp. 947-953, 2013.

[5] He J.and Zhang B.,"Soil Ionization Phenomenon around Grounding Electrode under Lightning Impulse", Asia-Pacific International Symposium and Exhibition on Electromagnetic Compatibility APEMC 2013, Engineers Australia:, pp. 94-98, Australia, 2013.

[6] Zeqing, S., Raghuveer M.R., and He J.,"Influence of the nature of impulse current propagation in soils on transient impedance characteristics", Electrical Insulation and Dielectric Phenomena, Vol.2, pp. 739-742, 2000.

[7] Yuki I., Shigeyasu M., Akiko, K., Hisatoshi, I. and Kunihiko H.,"ShackHartmann type laser wavefront sensor for measuring two-dimensional electron density distribution over extinguishing arc discharge", Journal of Physics D: Applied Physics, Vol. 45, no. 43, pp. 1-6, 2012.

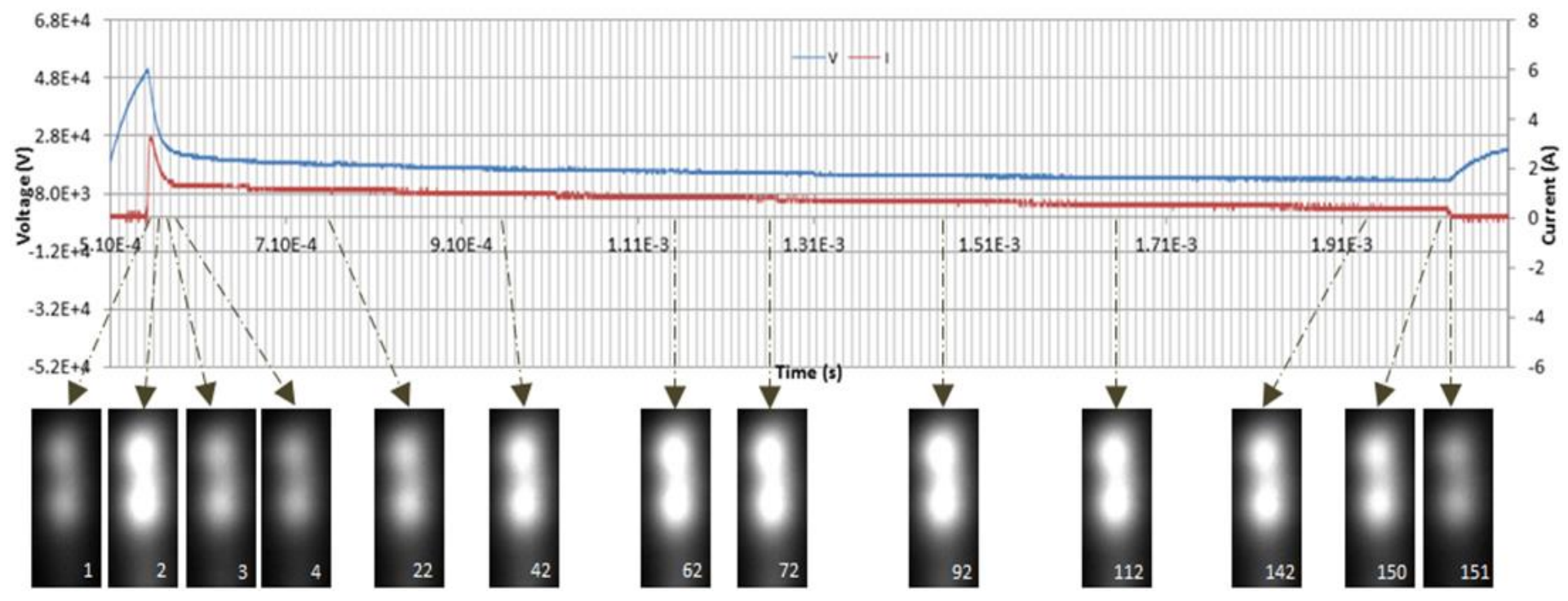

Figure 5. Synchronized video frames with voltage and current waveforms in the case of switching voltage 